\title{
Bilateral Thyroid Glands
}

National Cancer Institute

\section{Source}

National Cancer Institute. Bilateral Thyroid Glands. NCI Thesaurus. Code C160208.

Both the left and the right thyroid glands. 